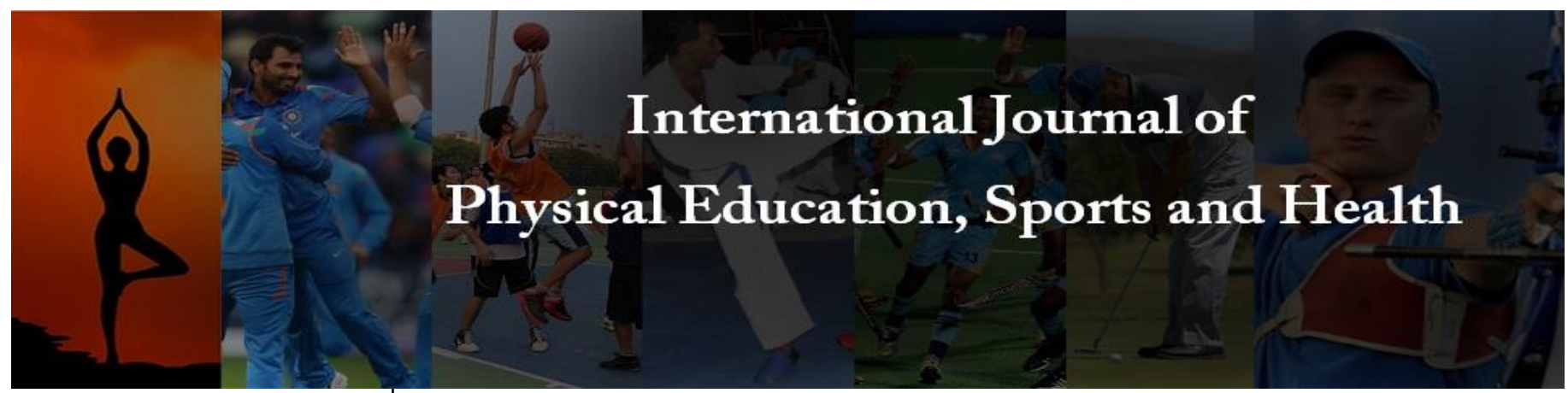

P-ISSN: 2394-1685

E-ISSN: 2394-1693

Impact Factor (ISRA): 5.38

IJPESH 2020; 7(1): 162-166

(C) 2020 IJPESH

www.kheljournal.com

Received: 22-11-2019

Accepted: 24-12-2019

Bernadett Kerteszne Nemet

Ph.D. Studies Orthopedic

Department, Orthopedic Clinic-

Semmelweis University,

Budapest, Hungary

TamasTerebessy

Orthopedic Department,

OrthopedicClinic-Semmelweis

University, Budapest, Hungary

Gyorgy Szoke

Orthopedic Department,

OrthopedicClinic-Semmelweis

University, Budapest, Hungary

Zoltan Bejek

Orthopedic Department,

OrthopedicClinic-Semmelweis

University, Budapest, Hungary

Corresponding Author:

Bernadett Kerteszne Nemet

Ph.D. Studies Orthopedic

Department, Orthopedic Clinic-

Semmelweis University,

Budapest, Hungary

\section{Kinesiology of kayaking in case of physical disablity with a special focus on footrest}

\author{
Bernadett Kerteszne Nemet, TamasTerebessy, Gyorgy Szoke and Zoltan \\ Bejek
}

Abstract

During kayaking, the whole body works in a perfect harmony. While the trunk is doing a rotation, flexion-extension helps to the upper limb to create a special cyclic paddle. The purpose of this study was to gain whether there were any significant differences between disabled and non-disabled kayakers with special focus on the use of footrest. Methods: Thirteen $(n=13)$ elite physical disabled athletes, eleven $(\mathrm{n}=11)$ elite non-disabled athletes and nine $(\mathrm{n}=9)$ athletes whose movements were artificially limited to imitate disabled conditions were measured. Results: Upper limb muscle activities were significantly different in the disabled group. In the disabled group, knee and trunk motions and muscle activities were also significantly different compared to the non-disabled group. Significant differences were found in performance, force and footrest use. Conclusions: Our results proved our assumption that motions and muscle activities of disabled and non-disabled athletes were significantly different.

Keywords: Physical disability, sport, kayak, kinesiology

\section{Introduction}

Sports and specifically kayak are not only for non-disabled athletes, but are examples of activities pursued by disabled people all over the world. Physical exercise is a very important part of life, especially in the case of disability ${ }^{[17]}$. Exercising supports both mental and physical well-being. Kayak is one of the sports suitable for physical disabled (later disabled) people. Sports can be a hobby or a profession for the disabled athlete. Para kayak is a sport recently adopted by disabled athletes, particularly its professional version, and it is not well described in terms of biomechanics. For this reason we chose to study para kayak. Para kayak became a formal event at the 2016 Paralympic Games. Kayak-canoe is a sport requires a specialised technique and intense physical effort ${ }^{[1,4]}$. The purpose of this study is to gain a better understand of the disabled person's kayaking motions and to probe whether there are significant differences between disabled and non-disabled kayakers with special focus on the use of footrest. A Weba sport kayak ergometer, surface electromyography (Siemens EMG), and a 3-dimensional Vicon (MX T40) camera system were used to record the data, and a combination MATLAB and MS Excel system was used to analyse all of them to get the results [5]. This specialised biomechanical method is a very effective and helpful analytical tool for other motions such as walking as well as surgical planning, orthopaedic follow-up and adaptive individual rehabilitation ${ }^{[2,9]}$. A scientific literature search shows the importance of para kayak method in rehabilitation and recreation ${ }^{[3]}$. In disability sports a level playing field is imperative, therefore the International Paracanoe Committee has created three categories for disabled athletes: Cluster 1, Cluster 2, and Cluster 3. These categories are not in direct correlation with levels or kinds of disability; instead, they indicate what kind of movements the athletes are able to carry out. Category Cluster3 is the most active and stable group ${ }^{[8]}$.

We hypothesised that the injury of the trunk and lower limbs does not hinder upper limb motions and muscle activities in the case of disability. Therefore, we did not expect significant differences between upper limb activity data from non-disabled and disabled athletes. We expected to find significant differences in the data describing the motions of the trunk, the lower extremities, and the footrest. 
A decrease in performance was hypothesised because of the lack of footrest use. We assumed significant differences both in force and performance.

It was important to determine the minimum and maximum limit values of the ranges of motion of the joints, muscle activities, and force applied to the footrest, in both nondisabled and disabled athletes $[10,13,14,15]$. The data of nondisabled athletes can serve as a starting point for the exploration of the expected movements of disabled athletes. We have also measured additional non-disabled athletes whose movements were artificially limited to imitate disabled conditions ("imitation disabled" group). Our study measured the accurate force of the lower limb by use of a built-in special dynamometer (devised locally in the laboratory) in the footrest during kayaking. Data from the literature shows that the contribution of lower limbs is a crucial point in kayaking, but due to the difficulty of measurement there is no accurate force data available ${ }^{[4,6,7,11,12,16]}$. It is possible that this force is not crucial for non-disabled athletes, but in disability it can be determined, because these athletes are not able to use their lower limbs for support via the footrest or only in a modified fashion. The supporting function of the lower limbs and the footrest helps the entire body to use the correct technique ${ }^{[1,12 \text {, }}$ $\left.{ }^{15}, 16\right]$ and when this function is missing the athlete's technique changes. That was the rationale for trying to learn more about this factor.

The aim of our study was to gain more information about the kinematic and muscle activities of disabled kayakers. These data may indicate what kind of motions can be expected from athletes with specific injuries.

The study was carried out in a special biomechanical laboratory at the Semmelweis University Orthopaedic Clinic in Budapest, Hungary, and supported by the University of Sport Sciences and the kayak-canoe department of the Hungarian National Sport Club.

\section{Materials and Methods}

A homogeneous group of athletes was chosen to guarantee more accurate results. All the athletes were professional male paddlers. Ethical approval has been obtained from the Ethics Committee of Hungary 14528-1/2019/EKU.

Thirteen $(n=13)$ elite physical disabled athletes (age range: 18-40 years, height range: $164-194 \mathrm{~cm}$, body mass range: $74-93 \mathrm{~kg})$, eleven $(\mathrm{n}=11)$ elite non-disabled athletes (age range: $18-40$ years, height range: $172-197 \mathrm{~cm}$, body mass range: $72-96 \mathrm{~kg}$ ) and nine $(\mathrm{n}=9)$ imitation disabled (age range: $18-40$ years, height range: $172-197 \mathrm{~cm}$, body mass range: $72-96 \mathrm{~kg}$ ) paddlers were measured on a kayak ergometer.

Befor the three-dimensional measurement the following arrangements were necessary:

Step 1: Anthropometric data have been recorded in the Vicon system: the length of the lower limbs (from the anterior superior iliac spine to the medial malleolus), width of the knee (from the medial side to the lateral side condyle of the tibia), width of the ankle (from the medial to the lateral side of the malleolus), distance of the shoulder and axilla(from the acromion to the axilla), the width of the elbow (from the medial to the lateral condyle of the humerus), width of the wrist (from the processus styloideus radii to the distal ulna) and the thickness of the palm ( $3^{\text {rd }}$ metacarpal joint) ${ }^{[5]}$. A special mechanical examination based on the International Paracanoe Committee (IPC) ${ }^{[8]}$ was used to check the disabled athletes' actual performance. In the imitation disabled group, the athlete's motions were artificially limited to imitate disabled conditions using special appliances.
Step 2: Light-reflecting markers were applied to anatomical points according to the Plug-in Gait protocol in Vicon system: acromion, suprasternal notch, distal part of sternum, upper part of humerus, lateral condyle of humerus, upper part of forearm, processus styloideus radii, distal part of ulna, $3^{\text {rd }}$ metacarpal, anterior superior iliac spine, posterior superior iliac spine, upper part of femur, lateral condyle of femur, upper part of tibia, and lateral malleolus. ${ }^{[4]}$ These markers were observable by the three-dimensional cameras.

Step 3. After fixing the markers on to the anatomical points and disinfecting the skin, electromyography electrodes were applied to the middle third of most important muscles both on the left and right side: latissimus dorsi muscle, anterior deltoid muscle, and pectoralis major muscle, biceps muscle of the arm, rectus femoris muscle, biceps femoris muscle, and external oblique abdominis muscle ${ }^{[6,7]}$.

The measurement process of the athletes were supervised by a physician and a coach. A Weba sport kayak ergometer, surface electromyography (EMG), and a 3-dimensional Vicon (MX T40) camera system were used to record the data. We used a Polar heart rate monitor to control the heart rate of the athletes during the investigation. Athletes were asked to complete a warm-up distance, then paddle a distance of 200 meters with $80 \%$ submaximal intensity, followed by cool down until their heart rate decreased to the prescribed range (minimum warm up heart rate, determined individually for each athlete). 200 meters is an official competition distance for both non-disabled and disabled athletes. All athletes were investigated during the same period, between 8:00 AM and 12:00 AM in all cases. If the disabled athlete competes wearing their prothesis or orthesis, they were instructed to wear the prothesis or orthesis during the investigation. Motions were observed with the help of the light-reflecting markers and the specialised 3-dimensional camera system. Muscle activity was also recorded in the Vicon system with the surface EMGs. Force and performance like the power of the footrest were recorded in the system of the kayak ergometer. All the measured values have been processed through MATLAB system. Welch was used through Excel system to evaluate the median values, providing a $95 \%$ confidence interval (CI).

\section{Results}

The average values were determined in non-disabled kayakers and the data were used to determine whether data measured in disabled athletes were significantly lower than the data of non-disabled athletes. (Table 1) In line with our basic assumption, range of motion of the upper limbs was not significantly different between disabled athletes and nondisabled athletes $(p \geq 0.05)$. However, muscle activities were significantly different in the disabled group compared to the non-disabled group $(p \leq 0.05)$. In the disabled group the knee joint and trunk motions and muscle activities were also significantly different compared to those in the non-disabled group $(p \leq 0.05)$. We also hypothesised that there would be a significant difference in performance and force, due to the involvement of the trunk or the lower limbs. For this reason, we expected that in the disabled group performance would decrease due to the lack of support via the footrest. This was underlined by measurements of athletes in the imitation disabled group, where the athletes were not able to use footrest in many cases and their performance was comparable to that of the disabled athletes. The imitation disabled group's data were analysed and significant differences were identified in all joint movements, except trunk and muscle activities. 
Table 1: Summary table about the average values: range of motions, muscle activity and force of footrest in 200m kayaking in case of abled and disabled athletes

\begin{tabular}{|c|c|c|}
\hline \multicolumn{3}{|c|}{ Summary Table } \\
\hline & Values & Values \\
\hline Muscle activity (v) & Disabled athletes & Abled athletes \\
\hline Right musculus deltoideus & 2,13 & 2,65 \\
\hline Right musculus latissimus dorsi & 1,64 & 3,25 \\
\hline Right musculus pectoralis major & 0,63 & 1,51 \\
\hline Right musculus biceps brachii & 1,09 & 2,87 \\
\hline Right nusculus obliques externus abdominis & 1,83 & 2,06 \\
\hline Right musculus rectus femoris & 0,05 & 0,34 \\
\hline Right musculus biceps femoris & 0,04 & 0,10 \\
\hline Left musculus deltoideus & 0,80 & 2,47 \\
\hline Left musculus latissimus dorsi & 0,63 & 2,95 \\
\hline Left musculus pectoralis major & 0,60 & 0,92 \\
\hline Left musculusc biceps brachii & 0,25 & 2,37 \\
\hline Left musculus obliques externus adbominis & 0,39 & 1,91 \\
\hline Left musculus rectus femoris & 0,02 & 0,45 \\
\hline Left musculus biceps femoris & 0,08 & 0,14 \\
\hline Joint movement(degree) & Disabled athletes & Abled athletes \\
\hline Right elbow flexion & 111,52 & 112,98 \\
\hline Left elbow flexion & 115,07 & 115,77 \\
\hline Right shoulder flexion & 84,77 & 85,68 \\
\hline Left shoulder flexion & 83,66 & 83,85 \\
\hline Trunk rotation & 0,07 & 12,98 \\
\hline Right knee flexion & 5,40 & 17,89 \\
\hline Left knee flexion & 4,24 & 22,04 \\
\hline & Disabled athletes & Abled athletes \\
\hline Left footrest(newton) & 96,97 & 208,11 \\
\hline Right footrest(newton) & 79,20 & 193,88 \\
\hline
\end{tabular}

The differences in performance (Figure 1), force applied to both footrests (Figure 2) and force (Figure 3) were significant $(p \leq 0.05)$. Accordingly, we can state that our hypothesis positing that kayaking motions in disabled and non-disabled athletes are significantly different was correct. The measurements from the imitation disabled group also reinforce our results.

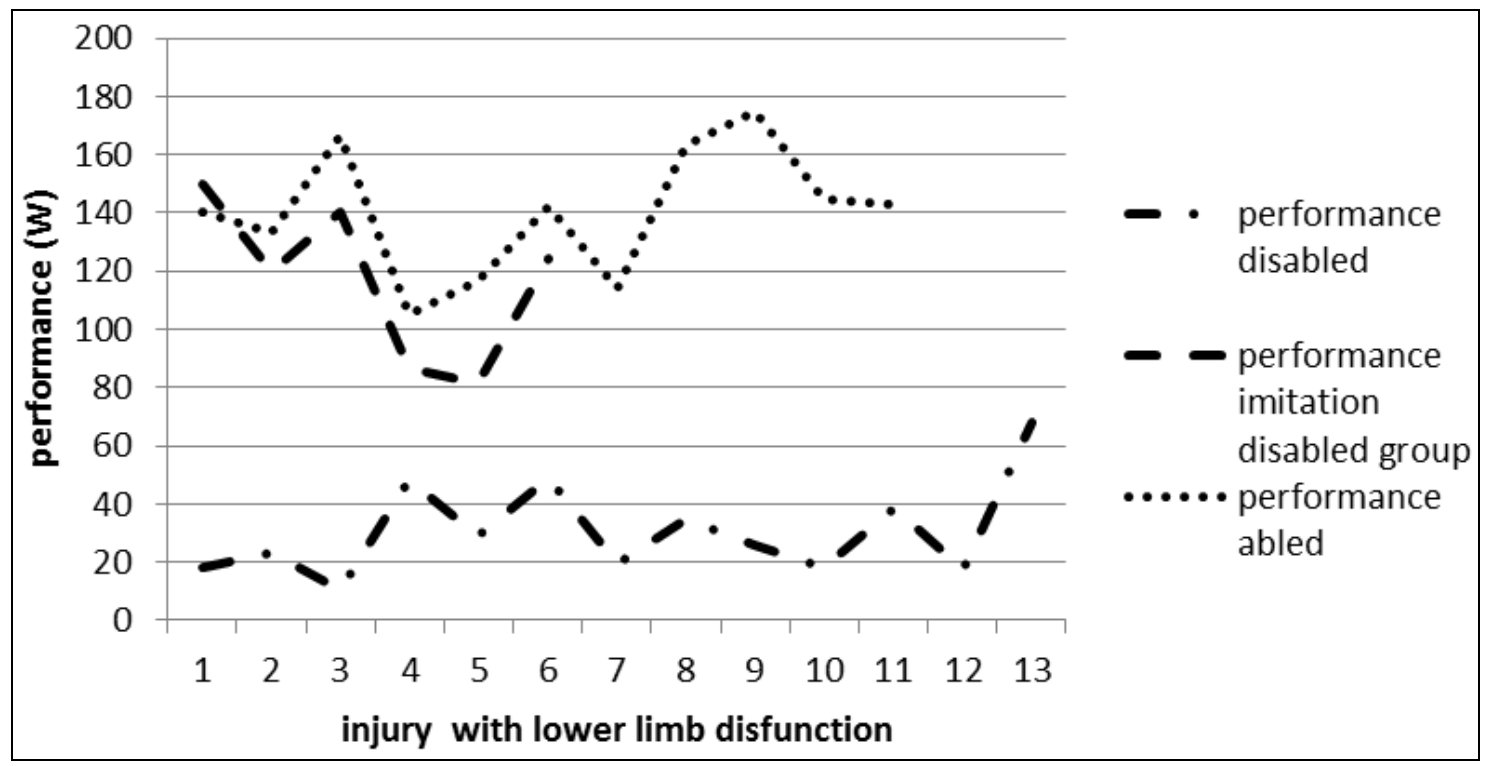

Fig 1: Comparison of the performance of professional male single kayakers in 200m kayaking. 


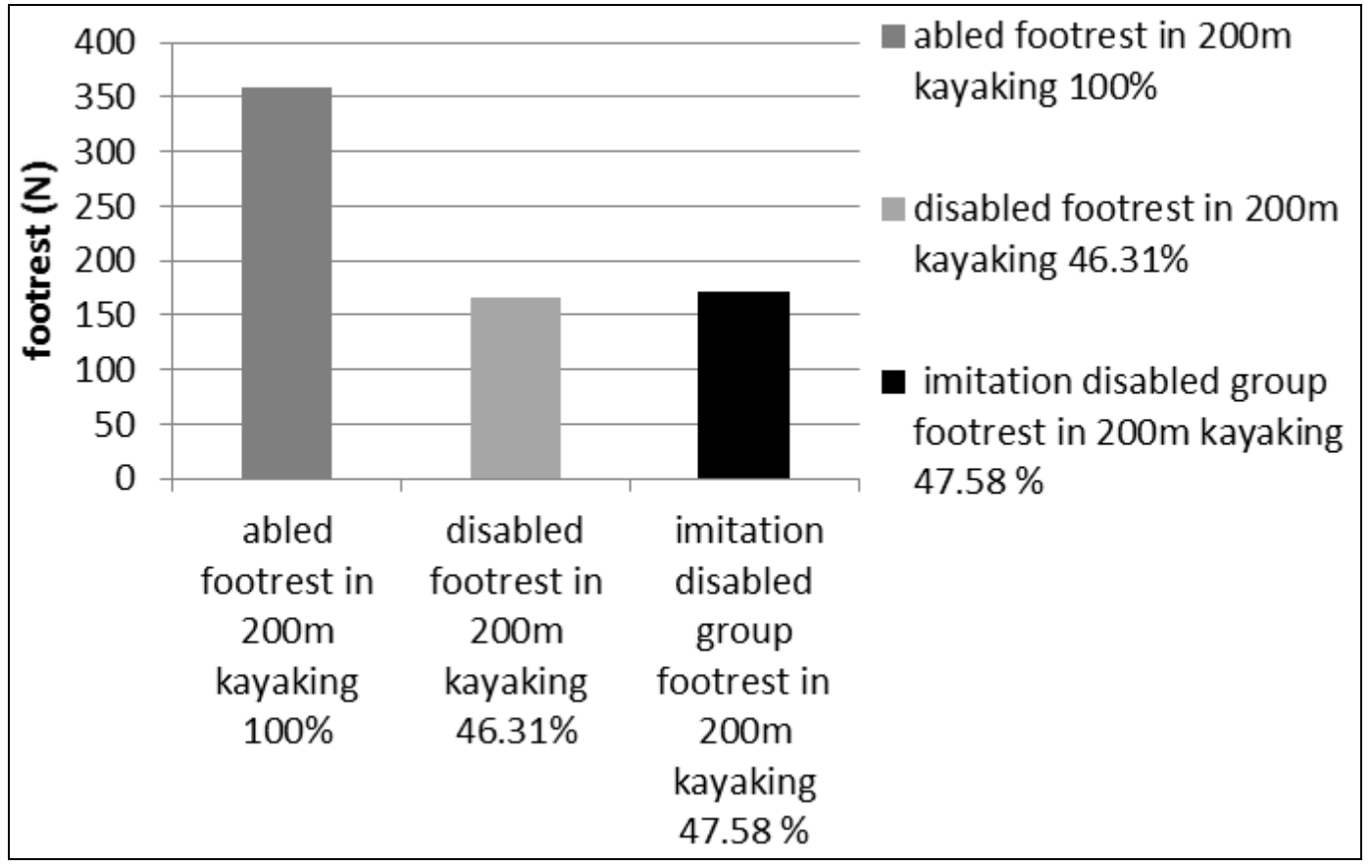

Fig 2: Comparison of the average footrest value in 200m kayaking by professional male single kayakers

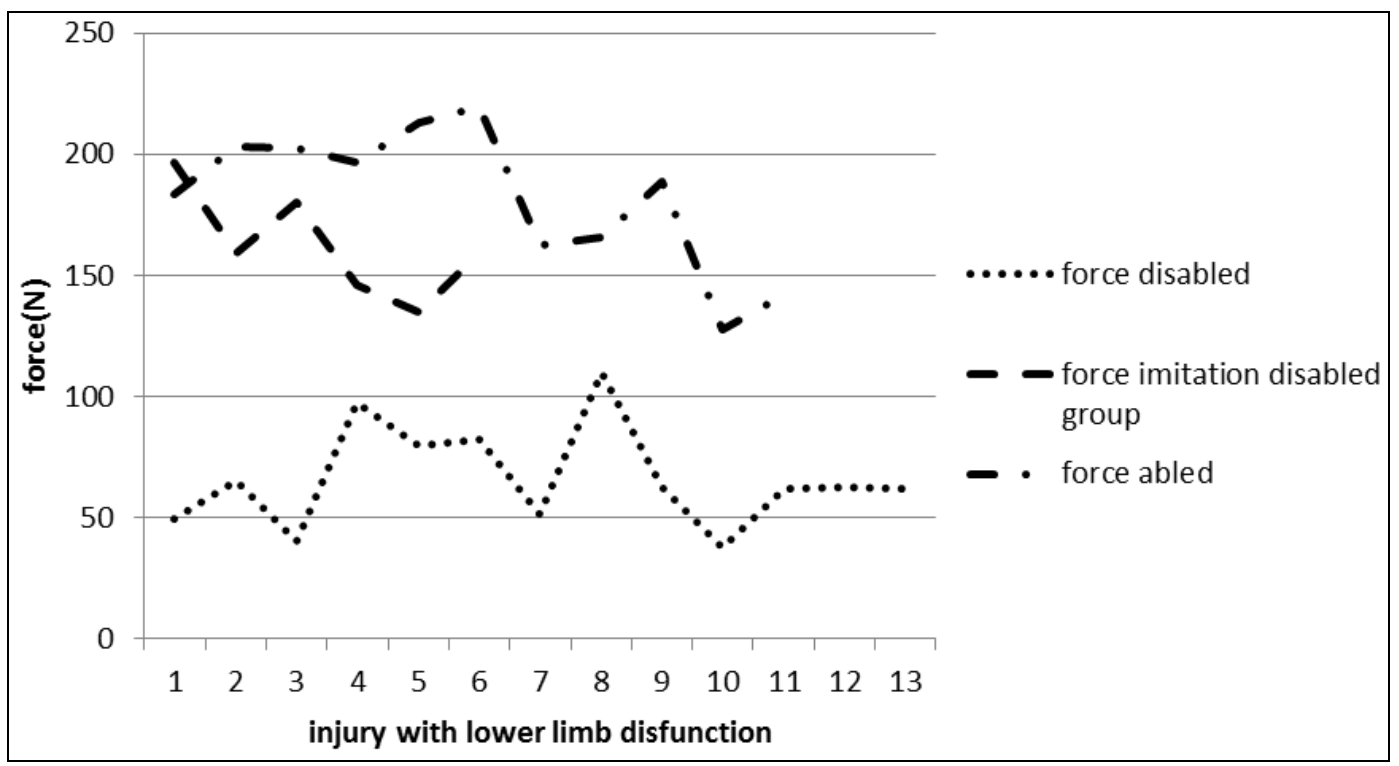

Fig 3: Comparison of the force by professional male single kayakers in 200m kayaking.

\section{Discussion}

In kayaking, non-disabled athletes are able to use the footrest fully, facilitating the correct execution of specialised technique, and they are able to output more force and longer stroke length and higher performance. In case of either symmetric or asymmetric disability, the athletes are unable to use the footrest fully, and as a consequence these athletes compensate their motions with their trunk and healthy upper limbs. These factors result in a shorter stroke length as well as lower force and performance. A special feature of our study was a built-in dynamometer in the footrest. With this dynamometer the force applied to the footrest could be measured numerically. The literature contains a profusion of data about the importance of kayaking in rehabilitation ${ }^{[4]}$, but there is not enough information about the use of footrest in kayaking. Although the importance of lower limbs and the footrest have already been investigated ${ }^{[16]}$, the force applied to the footrest has never been measured. Our study also demonstrated that the force applied to the footrest is also crucial in the case of disabled elite kayakers. The small investigational group supported us to be more accurate. In conclusion, we are confident that hobby and professional sports can be highly advantageous for disabled persons. In a lot of cases sports means life. Our study provided novel information about how an athlete compensates to be able to do kayaking with a physical disability, and we obtained new data on differences in performance and force. The results indicate the need for further investigations of the correlations we have uncovered, and hopefully we can learn more about the motion of disabled athletes in this sport. Our results greatly contribute to devising appropriate training methods to avoid sports injuries a major issue in professional sports. Injuries can mean long periods of absence from the racing calendar and they should be avoidable. They can also bring about both physical and emotional problems. Data can indicate the way to more specialised and individually tailored training methods for coaches and athletes. Better training methods result in better performance for both non-disabled and disabled paddlers. As para sports show a growing trend, scientific research should focus more on this area. A deeper 
understanding of para sports can ensure better professional training for para-athletes, although our hope is that the results of this study can provide motivation to all disabled persons for any kind of sporting activities and not only for professional sports. In conclusion, we are confident that hobby and professional sports can be highly advantageous for disabled persons

\section{Acknowledgement}

Bernadett Kertesz Nemet as the corresponding author, declare, that the manuscript was controlled and assessed by co-authors. This manuscript is not in buplication progression by other journal. The investigation did not receive financial support from any sponsor. The authors would like to express a great debt of gratitude to the participants of the study. This research was performed in the biomechanical laboratory of the Orthopaedic Clinic of Semmelweis University, which provided facilities and appropriate conditions for the study with MXT three dimensional camera system. Ethical approval has been obtained from the Ethics Committee of Hungary 14528-1/2019/EKU. I'm truly grateful for my co-author's assistance and for the opportunity to work in the laboratory.

\section{Conflicts of Interest}

The authors declare that they have no conflicts of interest. The manuscript was controlled and assessed by all authors. The authors declare that they have no known competing financial interests or personal relationships that could have appeared to influence the work reported in this paper.

\section{References}

1. Begon M, Colloud F, Sardain P. Lower limb contribution in kayak performance modelling, simulation and analysis. Multibody SYST Dyn. 2010; 23:387-400.

2. Bejek Z, Paróczai R, Illyés Á, Kocsis L, Kiss RM. Gait parameters of patients with osteoarthritis of the knee joint. Facta Universitatis: Series Physical Education and Sport. 2006; 4(1):9-16.

3. Bjerkefors A, Thorstensson A. Effects of Kayak Ergometer Training on Motor Performance in Paraplegics. Int J of Sports Medi. 2006; 27:824-9.

4. Brown MB, Lauder M, Dyson R. Activation and contribution of trunk and leg musculature to force production during on-water sprint kayak performance. International Symposium on Biomechanics in Sports: Conference Proceedings Archive. 2010; 28:1-4.

5. Dempter WT. Space requirements of the seated operator. Wright-Patterson Air Force Base, OH.WADC Technical Report, 1995, 55-159.

6. Fleming N, Donne B, Fletcher D et al. Effect of kayak ergometer elastic tension on upper limb EMG activity and 3D kinematics. J Sports Sci and Med. 2012; 11:430437.

7. Hibbs AE, Thompson KG, French DN, et al. Peak and average rectified EMG measures: Which method of data reduction should be used for assessing core training exercises? Electromyography Kines. 2011; 21:102-111.

8. https://www.canoeichf.com/classification

9. Illyés Á, Bejek Z, Szlávik I, Paróczai R, Kiss RM. Threedimensional gait analysis after unilateral cemented total hip arthroplasthy. Facta Universitatis: Series Physical Education and Sport. 2006; 4(1):27-34.

10. Lovell G, Lauder M. Bilateral strength comparisons among injured and non-injured competitive flatwater kayakers. J Sport Rehabil. 2001; 10:3-10.
11. Limonta E, Squadrone $\mathrm{R}$, Rodano $\mathrm{R}$ et al. Tri dimensional kinematic analysis on a kayaking simulator: key factors to successful performance. J Sport Health Sci. 2010; 6:27-34.

12. McDonnell LK, Hume PA, Nolte V. An observational model for biomechanical assessment of sprint kayaking technique. Sports Biomech. 2012; 11:507-523.

13. McKean MR, Burkett B. The relationship between joint range of motion, muscular strength, and race time for sub-elite flat water kayakers. J Sci Med. Sport 2010; 13:537-542.

14. Michael JS, KB Rooney, Smith RM. The dynamics of elite paddling on a kayak simulator. J Sports Sci. 2012; 30:661-668.

15. Michael JS, Smith R, Rooney KB. Determinants of kayak paddling performance. Sports Biomech. 2009; 8:167-179.

16. Nilsson JE, Rosdah HG. 1. Contribution of Leg-Muscle Forces to Paddle Force and Kayak Speed during Maximal-Effort Flat-Water Paddling. Int J Sports Physiol. 2016; 11:22-27.

17. Shoga D. The social construction of disability: The impact of statistics and technology. Adapt Phys Act Q. 1998; 15:269-277. 